\title{
Obesity resistance and enhanced glucose metabolism in mice transplanted with white adipose tissue lacking acyl CoA:diacylglycerol acyltransferase 1
}

\author{
Hubert C. Chen, ${ }^{1,2,3}$ Dalan R. Jensen, ${ }^{4}$ Heather M. Myers, ${ }^{1}$ Robert H. Eckel,${ }^{4}$ and \\ Robert V. Farese, Jr. ${ }^{1,2,3}$ \\ ${ }^{1}$ Gladstone Institute of Cardiovascular Disease, San Francisco, California, USA \\ ${ }^{2}$ Cardiovascular Research Institute, and \\ ${ }^{3}$ Department of Medicine, University of California, San Francisco, San Francisco, California, USA \\ ${ }^{4}$ University of Colorado Health Sciences Center, Denver, Colorado, USA
}

\begin{abstract}
Recent studies have identified the white adipose tissue (WAT) as an important endocrine organ that regulates energy and glucose metabolism via a number of secreted factors. Mice lacking acyl CoA:diacylglycerol acyltransferase 1 (DGAT1), a key enzyme in mammalian triglyceride synthesis, are protected against diet-induced obesity and glucose intolerance because of increased energy expenditure and enhanced insulin sensitivity. Because DGAT1 is highly expressed in WAT, we hypothesized that DGAT1 deficiency affects the expression of adipocyte-derived factors that regulate energy and glucose metabolism. Here we show that the transplantation of DGAT1-deficient WAT decreases adiposity and enhances glucose disposal in wild-type mice. Analysis of DGAT1-deficient WAT revealed a twofold increase in the expression of adiponectin, a molecule that enhances fatty acid oxidation and insulin sensitivity, and this increase may account in part for the transplantation-induced metabolic changes. Our results highlight the importance of the endocrine function of WAT and suggest that an alteration in this function contributes to the increased energy expenditure and insulin sensitivity in DGAT1-deficient mice.
\end{abstract}

J. Clin. Invest. 111:1715-1722 (2003). doi:10.1172/JCI200315859.

\section{Introduction}

Recent studies have altered conceptions of the adipose tissue as an inert tissue that serves merely to store energy. Instead, it plays a vital role in regulating energy and glucose homeostasis $(1,2)$, in part by secreting factors such as leptin (3), adiponectin $(4,5)$, TNF- $\alpha(6)$, and resistin (7) that modulate the storage, mobilization, and utilization of lipids and glucose. The importance of adipose tissue in normal physiology is demonstrated by the insulin resistance and abnormal lipid metabolism in mice that lack fat $(8,9)$ or that do not secrete adipocytederived factors such as leptin (3). In addition, the transplantation of normal adipose tissue into A-ZIP/F1 fatless (lipoatrophic) mice improves their metabolic function (10), in part by restoring the expression of adipocyte-derived factors.

A number of genetic alterations in mice result in a leanness phenotype, and several of these models also have increased insulin sensitivity (reviewed in ref. 11).

Received for publication May 3, 2002, and accepted in revised form April 1, 2003.

Address correspondence to: Robert V. Farese, Jr., Gladstone Institute of Cardiovascular Disease, PO Box 419100, San Francisco, California 94141-9100, USA. Phone: (415) 826-7500; Fax: (415) 285-5632; E-mail: bfarese@gladstone.ucsf.edu.

Conflict of interest: The authors have declared that no conflict of interest exists.

Nonstandard abbreviations used: acyl CoA:diacylglycerol acyltransferase 1 (DGAT1); white adipose tissue (WAT).
One such model is mice that lack acyl CoA:diacylglycerol acyltransferase 1 (DGAT1) (12), one of two known enzymes that catalyze the final step in mammalian triglyceride synthesis $(13,14)$. DGAT1-deficient (Dgat1 $\left.1^{-/}\right)$mice synthesize triglycerides through the actions of DGAT2 (15) and possibly other mechanisms (14). However, these mice are protected against dietinduced obesity and insulin resistance (12) because of increased energy expenditure (12) and enhanced insulin sensitivity (16). Because DGAT1 is highly expressed in the white adipose tissue (WAT) (13), we hypothesized that DGAT1 deficiency affects the endocrine function of WAT and alters the secretion of adipocyte-derived factors that act systemically to increase energy expenditure and insulin sensitivity.

To test this hypothesis, we transplanted WAT from Dgat $1^{-/-}$mice into mice prone to obesity and insulin resistance and assessed its effects on glucose disposal and the response to a high-fat diet. We also examined the expression of several adipocyte-derived factors that regulate energy and glucose metabolism in Dgat1 $1^{-/}$ WAT. Our results indicate that DGAT1 deficiency alters the endocrine function of WAT and suggest that this alteration contributes to the increased energy expenditure and insulin sensitivity in $D g a t 1^{-/-}$mice.

\section{Methods}

Mice. Dgat $1^{-/-}$mice ( $99 \%$ C57BL/6, 1\% 129/SvJae background) were generated previously (12). Wild-type 
$\left(\right.$ Dgat $\left.1^{+/+}\right)$, Agouti yellow $\left(A^{Y} / a\right)$, and heterozygous leptindeficient $(o b /+)$ mice (all on the C57BL/ 6 background) were from The Jackson Laboratory (Bar Harbor, Maine, USA). $A^{Y} / a$ mice are insulin- and leptin-resistant (17), reflecting the antagonism of melanocortin receptors (18), whereas leptin-deficient $(o b / o b)$ mice are obese and diabetic because of leptin deficiency (3). Genotyping was performed as previously described $(12,19)$. Mice were housed in a pathogen-free barrier facility (12-hour light/12-hour dark cycle) and fed rodent chow (Ralston Purina Co., St. Louis, Missouri, USA). For high-fat diet experiments, mice were fed a Western-type diet (21\% fat by weight; Harlan Teklad, Madison, Wisconsin, USA). All experiments were approved by the Committee on Animal Research of the University of California, San Francisco.

Fat transplantation. Fat transplantation was performed as previously described (10). Donor and recipient mice were age- and sex-matched to minimize rejection. Reproductive fat pad $(500 \mathrm{mg})$ from the donor was inserted into the subcutaneous space on the back of the recipient. The amount of WAT transplanted represented about $30 \%$ of the total fat pad weight in chow-fed C57BL/ 6 mice and less than $10 \%$ of the total fat pad weight in $A^{Y} / a$ and $o b / o b$ mice. At the end of experiments, the grafts were evaluated visually and fixed for histological analysis.

Fat pad measurement. Unless stated otherwise, reproductive, inguinal, mesenteric, and perirenal fat pads were isolated and weighed 2 weeks after transplantation for chow experiments and 10 weeks after transplantation for high-fat experiments.

Serum protein measurements. Leptin and TNF- $\alpha$ levels were measured with enzyme immunoassay kits (R\&D Systems Inc., Minneapolis, Minnesota, USA). Adiponectin levels were measured by AniLytics (Gaithersburg, Maryland, USA).

Triglycerides, protein, and lean body mass measurements. For mice fed a high-fat diet for 5-6 weeks, total body triglycerides and muscle triglycerides were measured as previously described (12). Muscle samples comprised the quadriceps, soleus, and gastrocnemius muscles from the hind legs. Total body protein was measured with a colorimetric kit (Bio-Rad Laboratories Inc., Hercules, California, USA). For mice fed a high-fat diet for 12 weeks, body triglycerides and lean body mass were measured by dual-energy x-ray absorptiometry (GE Medical Systems; General Electric Co., Waukesha, Wisconsin, USA).

Energy metabolism studies. Food consumption and energy expenditure were measured in metabolic chambers as previously described (12). Experiments were performed after 12 weeks of high-fat feeding.

Glucose metabolism studies. Glucose (1 $\mathrm{g} / \mathrm{kg}$ body weight) or bovine insulin ( $1 \mathrm{U} / \mathrm{kg}$ body weight; Sigma Chemical Co., St. Louis, Missouri, USA) was injected intraperitoneally 1 hour after fasting, and glucose concentrations were measured with a glucometer (Accu-chek; Roche Diagnostics Corp., Indianapolis, Indiana, USA). Experiments were performed 1 week after transplantation.

\section{Table 1}

Real-time PCR primer and probe sequences

\begin{tabular}{|c|c|c|}
\hline Gene & $\begin{array}{l}\text { rimer pair } \\
\text { or probe }\end{array}$ & Sequence \\
\hline \multirow[t]{3}{*}{ Actin } & $5^{\prime}$ & 5'-САTСТTGGССТСАСТGTCСА-3' \\
\hline & $3^{\prime}$ & 5'-GGGCCGGACTCATCGTACT-3' \\
\hline & Probe & 5'-CTTCCAGCAGATGTGGATCAGCAAGC-3' \\
\hline \multirow[t]{3}{*}{ Adiponectin } & $5^{\prime}$ & 5'-TGTTGGAATGACAGGAGCTGAA-3' \\
\hline & $3^{\prime}$ & 5'-CACACTGAACGCTGAGCGATAC-3' \\
\hline & Probe & 5'-TCCCCGGAACCCCTGGCAG-3' \\
\hline \multirow[t]{3}{*}{ Resistin } & $5^{\prime}$ & 5'-ACAGGAGCTAATACCCAGAACTGAGT-3' \\
\hline & $3^{\prime}$ & $5^{\prime}$-GACACAGTGGCATGCTGGAG-3' \\
\hline & Probe & 5'-TCСTाTCTTCСТTGTСССТGAACTGCTG-3' \\
\hline \multirow[t]{3}{*}{ TNF- $\alpha$} & $5^{\prime}$ & 5'-CAGGCAGGAAACCCTTGAGA-3' \\
\hline & $3^{\prime}$ & $5^{\prime}$-AGCCTCCAAGCCCAGAAACT-3' \\
\hline & Probe & 5'-CGCATGTGCAGGGCACAACTCC-3' \\
\hline
\end{tabular}

Real-time PCR. Real-time PCR was performed as previously described (20). Primer and probe sequences (Table 1) were selected with Primer Express (Perkin-Elmer Applied Biosystems, Foster City, California, USA).

Leptin administration. Mice were infused with recombinant murine leptin (a gift from Amgen Inc., Thousand Oaks, California, USA) for 5 days with a microosmotic pump (Alza model 1002; DURECT Corp., Cupertino, California, USA) inserted subcutaneously into the interscapular region.

Tissue culture. Reproductive fat pads $(500 \mathrm{mg})$ from chow-fed male $A^{Y} / a D g a t 1^{+/+}$and $A^{Y} / a$ Dgat $1^{-/-}$mice were sliced into 50-mg pieces, washed twice with PBS, placed in DMEM (Invitrogen Corp., Carlsbad, California, USA) supplemented with 1\% FBS (HyClone Laboratories, Logan, Utah, USA), and incubated at $37^{\circ} \mathrm{C}$ and $5 \% \mathrm{CO}_{2}$. The conditioned media were collected after 10-12 hours. Total protein was measured with a colorimetric kit (Bio-Rad Laboratories Inc.), FFAs were measured as previously described (12), leptin and TNF- $\alpha$ were measured with enzyme immunoassay kits (R\&D Systems Inc.), and adiponectin was measured with an ELISA kit (B-Bridge International Inc., San Jose, California, USA). These factors were also measured in growth media not conditioned by adipose tissue, and the basal levels were subtracted from the reported results.

Immunoblots. Serum samples $(1 \mu \mathrm{l})$ were loaded onto a $10 \%$ polyacrylamide gel, transferred to a nitrocellulose membrane, and incubated with a polyclonal primary antibody that recognizes the globular head region of murine adiponectin (a gift from Genset Corp., San Diego, California, USA). Binding was detected by an anti-IgG antibody-peroxidase conjugate (Santa Cruz Biotechnology Inc., Santa Cruz, California, USA) and enhanced chemiluminescence (Amersham Pharmacia Biotech, Buckinghamshire, United Kingdom).

Statistical methods. Data are shown as mean \pm SD unless stated otherwise. Measurements were compared with the two-tailed $t$ test or Mann-Whitney rank-sum test. Energy expenditure and lean body mass measurements were analyzed by linear regression. Weight curves were 


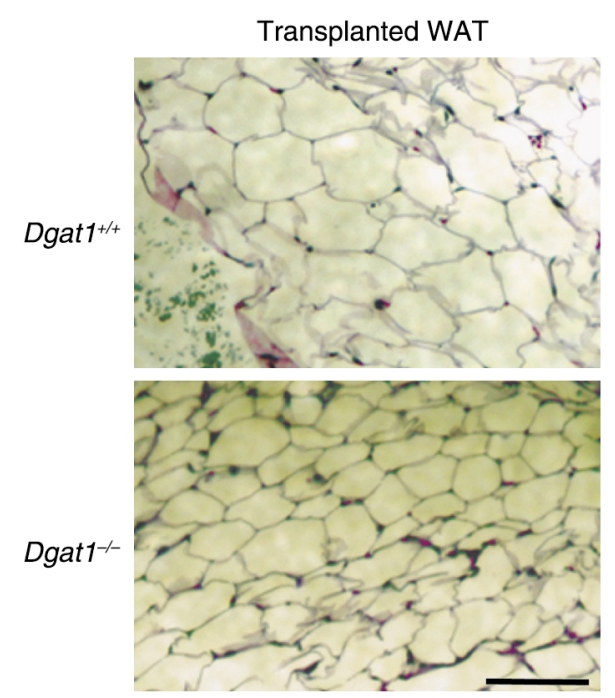

Figure 1

Histology of transplanted Dgat $1^{+/+}$and Dgat $1^{-/-}$WAT. Representative results are shown. Bar, $100 \mu \mathrm{m}$.

compared with repeated-measures ANOVA, and serum adiponectin and TNF- $\alpha$ results were compared with ANOVA. Both comparisons were followed by post hoc Tukey-Kramer test, if appropriate, to determine the effect of genotype.

\section{Results}

Fat transplantation. We transplanted WAT from Dgat1 $1^{-/}$ mice into $D$ gat $1^{+/+}$mice $\left(\right.$WAT $^{\text {Dgat }} 1^{-/} \rightarrow D$ gat $1^{+/+}$). We also transplanted a similar amount of WAT from $D g a t 1^{+/+}$ mice into Dgat $^{+/+}$mice as controls $\left(\mathrm{WAT}^{\text {Dgat }}\right.$ 1/+ $^{++} \rightarrow$ $\left.D g a t 1^{+/+}\right)$. Ninety mice received transplants during the study, and 72 had viable grafts, as judged by visual inspection, at sacrifice. The transplanted fat pads retained their characteristic histological appearance (16); that is, Dgat $1^{-/}$WAT contained smaller adipocytes than Dgat1 $1^{+/+}$WAT (Figure 1). The transplanted fat pads decreased slightly in weight 20 weeks after the procedure $\left(410 \pm 36 \mathrm{mg}\right.$ for $D g a t 1^{-/-}$WAT and $423 \pm 33 \mathrm{mg}$ for Dgat $1^{+/+}$WAT).

Resistance to diet-induced obesity in wild-type mice transplanted with Dgat $1^{-/-}$WAT. WAT Wgat $^{1^{-/}} \rightarrow$ Dgat $1^{+/+}$mice gained less weight than WAT Dgat $^{1^{++}} \rightarrow D g a t 1^{+/+}$mice in response to a Western-style high-fat diet (Figure 2a). The difference in weight curves became apparent after 4 weeks of high-fat feeding. WAT $^{\text {Dgat }^{-/-}} \rightarrow$ Dgat $1^{+/+}$mice with nonviable grafts at sacrifice had weight curves similar to those of $\mathrm{WAT}^{\text {Dgat }^{+/+}} \rightarrow$ Dgat $1^{+/+}$mice $(46.2 \pm 2.3$ vs. $47.5 \pm 1.6 \mathrm{~g}, P>0.05)$, indicating that obesity resistance in these mice required viable grafts. WAT ${ }^{\text {grat }}{ }^{-/-} \rightarrow$ Dgat $^{+/+}$mice with viable grafts also had an approximately $50 \%$ reduction in total fat pad content when fed either a chow or a high-fat diet (Figure $2 \mathrm{~b}$ ). In contrast, transplantation of $D g a t 1^{+/+}$WAT into Dgat1 $1^{-/}$mice did not affect their resistance to diet-induced obesity (Figure 2c) or total fat pad content (Figure 2d).
To further characterize the effects of transplanted Dgat1 $1^{-/}$WAT on obesity resistance, we measured serum leptin levels as a marker of adiposity. WAT $^{\text {Dgat } 1^{-/}} \rightarrow$ $D_{\text {gat }} 1^{+/+}$and WAT $^{\text {Dgat }}{ }^{+/+} \rightarrow$ Dgat $1^{+/+}$mice had similar serum leptin levels after 2 weeks of high-fat feeding (Figure 3a). However, after 5 weeks of high-fat feeding, WAT $^{\text {ggat }} 1^{-/-} \rightarrow$ Dgat $1^{+/+}$mice had lower serum leptin levels than $\mathrm{WAT}^{\text {Dgat }}{ }^{+/+} \rightarrow D$ gat $1^{+/+}$mice. This difference in serum leptin levels corresponded to the divergence in weight curves. WAT Dgat $^{-/} \rightarrow$ Dgat $1^{+/+}$mice had lower levels of body triglycerides (Figure 3b) than WAT $D$ gat $^{+/+} \rightarrow$ Dgat $1^{+/+}$mice, after either 5 or 12 weeks of high-fat feeding. WAT Dgat $1^{-/-} \rightarrow D$ gat $1^{+/+}$mice also had decreased levels of muscle triglycerides (Figure 3c) after 5 weeks of high-fat feeding. In contrast, WAT ${ }^{\text {Dgat }}{ }^{-/-} \rightarrow$ Dgat1 $1^{+++}$and WAT $^{\text {Dgat }}{ }^{+/+} \rightarrow$ Dgat $1^{+/+}$mice had similar amounts of total body protein or lean body mass (Figure 3d). These results indicate that transplanted Dgat $1^{-/-}$ WAT decreased adiposity without affecting lean body mass in the recipient mice.

To investigate the mechanism for this resistance to diet-induced obesity in $\mathrm{WAT}^{\text {Dgat }} 1^{-/} \rightarrow$ Dgat $^{+/+}$mice, we fasted the mice for 16 hours and used the fasting-associated weight loss as a surrogate of energy expenditure. WAT $_{\text {Dgat } 1^{-}} \rightarrow D$ gat $1^{+/+}$mice lost more weight than WAT $^{\text {Dgat }}{ }^{+/+} \rightarrow D$ gat $1^{+/+}$mice after the fast $(3.4 \pm 0.1 \mathrm{vs}$. $2.9 \pm 0.3 \mathrm{~g}, n=4, P<0.05)$ and ate more during the
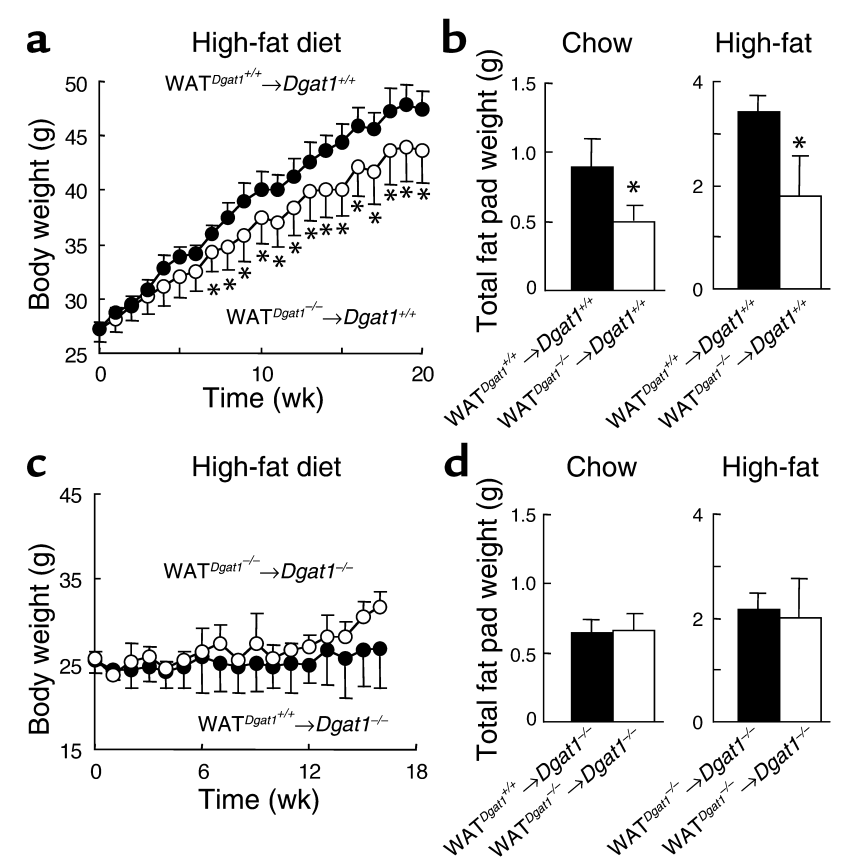

\section{Figure 2}

Effects of WAT transplantation on body weight and total fat pad content. (a) Decreased weight gain in WAT Dgat $^{-/-} \rightarrow$ Dgat $1^{+/+}$mice fed a high-fat diet. $n=8-9$ male mice per group. (b) Decreased total fat pad content (excluding transplanted fat pads) in WAT Wgat $^{1^{-/}} \rightarrow$ Dgat $^{+/+}$ mice. $n=4-6$ male mice per group. (c) Lack of effect of transplanted Dgat $1^{+/+}$WAT on obesity resistance of Dgat $1^{-/-}$mice. $n=3-4$ female mice per group. (d) Lack of effect of transplanted Dgat $1^{+/+}$WAT on total fat pad content in Dgat $1^{-/-}$mice. $n=4$ male mice per group. ${ }^{*} P<0.05$. 

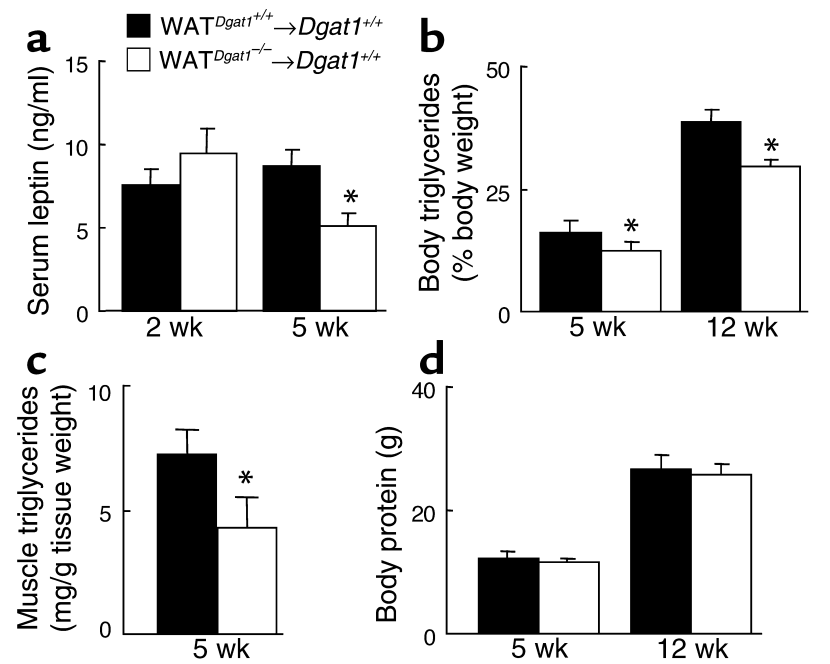

Figure 3

Effects of transplanted Dgat $1^{-/-}$WAT on adiposity in mice fed a highfat diet. (a) Decreased serum leptin levels in WATDgat ${ }^{-/-} \rightarrow$ Dgat $1^{+/+}$ mice. $n=4-5$ male mice per group. (b) Decreased body triglyceride content in WAT Dgat $^{-/-} \rightarrow$ Dgat $1^{+/+}$mice. $n=4$ male mice per group. (c) Decreased muscle triglyceride content in WAT Dgat $1^{-/-} \rightarrow$ Dgat $1^{+/+}$ mice. $n=3$ male mice per group. (d) Similar body-protein contents in WAT Wat $^{1^{-/}} \rightarrow$ Dgat $^{1^{+/+}}$and WAT ${ }^{\text {gat } 1^{+/+}} \rightarrow$ Dgat $1^{+/+}$mice. $n=4$ male mice per group. Mice were fed a high-fat diet for 2, 5, or 12 weeks. ${ }^{*} P<0.05$.

refeeding $(6.7 \pm 0.5$ vs. $6.0 \pm 0.1 \mathrm{~g} / 24 \mathrm{~h}, P<0.05)$. The enhanced weight loss in WAT ${ }^{\text {ggat }}{ }^{-1} \rightarrow D_{\text {gat }}{ }^{+/+}$mice suggested an increase in energy expenditure. To further investigate this, we conducted energy-balance studies on transplanted mice fed a high-fat diet. When adjusted for total body weight, WAT ${ }^{\text {Dgat } 1^{-/}} \rightarrow$ Dgat $1^{+/+}$and WAT $^{\text {Dgat1 }}{ }^{+/+} \rightarrow$ Dgat $1^{+/+}$mice consumed similar amounts of calories (Figure 4a); however, WAT $^{\text {Dgat }}{ }^{-/-} \rightarrow D$ gat $1^{+/+}$ mice had increased energy expenditure. Plotting of the daily metabolic rate versus lean body mass for each mouse revealed that values for WAT $^{\text {Dgat }}{ }^{-/} \rightarrow$ Dgat $1^{+/+}$ mice were on or above the regression line for WAT $^{\text {Dgat }}{ }^{+/+} \rightarrow D$ gat $1^{+/+}$mice (Figure $4 \mathrm{~b}$ ), consistent with increased energy expenditure.

Enhanced glucose disposal in wild-type mice transplanted with Dgat1 $1^{--}$WAT. To assess the effects of the WAT transplantation on glucose metabolism, we conducted glucose and insulin tolerance tests. Chow-fed $\mathrm{WAT}^{\text {Dgat }} 1^{-/} \rightarrow$ Dgat $1^{+/+}$mice had lower blood glucose concentrations than chow-fed WAT Dgat $^{+/+} \rightarrow$ Dgat $1^{+/+}$mice after either a glucose load (Figure 5a) or an insulin injection (Figure $5 \mathrm{~b}$ ), suggesting increased insulin sensitivity in Dgat1 $1^{+/+}$ mice transplanted with Dgat1 $1^{--}$WAT. In contrast, WAT $^{\text {Dgat }}{ }^{+/+} \rightarrow$ Dgat $1^{-/}$and WAT ${ }^{\text {Dgat } 1-/-} \rightarrow D$ gat $1^{-/-}$mice had similar glucose concentrations after either a glucose load (Figure 5c) or an insulin injection (Figure 5d), suggesting that $D$ gat $1^{+/+}$WAT did not affect the glucose disposal of $D$ gat $1^{-/-}$mice.

Transplantation of Dgat1 $1^{-/-}$WAT decreases adiposity and enhances glucose disposal in Agoutiyellow but not ob/ob mice. We performed WAT transplantation experiments in two genetic models of obesity and insulin resistance, the Agouti yellow $\left(A^{Y} / a\right)$ and the leptin-deficient $(o b / o b)$ mice. We had introduced DGAT1 deficiency into both models by genetic crosses in previous studies $(16,20)$. One week after transplantation, $A^{Y} / a$ Dgat $1^{+/+}$mice transplanted with $A^{Y} / a$ Dgat $1^{-/-}$WAT (WAT ${ }^{A^{Y} / a D g a t 1^{-/}} \rightarrow$ $A^{Y} / a D g a t 1^{+/+}$) and control mice $\left(\right.$WAT $^{A^{Y} / a D g a t 1^{+/+}} \rightarrow A^{Y} / a$ Dgat $\left.1^{++}\right)$had similar mean body weights $(32.7 \pm 1.2 \mathrm{vs}$. $33.6 \pm 2.4 \mathrm{~g}, n=4-5, P>0.05)$. WAT $A^{A^{Y} / a D g a t 1^{-/-}} \rightarrow A^{Y} / a$ Dgat $1^{+/+}$mice, however, had lower blood glucose concentrations than control mice during both glucose tolerance (Figure 6a) and insulin tolerance (Figure 6b) tests, suggesting increased insulin sensitivity. After 6 weeks of a high-fat diet, $\mathrm{WAT}^{A^{Y} / a D g a t 1^{-/}} \rightarrow A^{Y} / a$ Dgat $1^{+/+}$ mice had a lower mean body weight ( $47.0 \pm 0.9$ vs. $50.3 \pm 1.9 \mathrm{~g}, n=4, P<0.05)$ and total fat pad content ( $8.7 \% \pm 0.6 \%$ vs. $9.6 \% \pm 0.1 \%$ of total body weight, $n=3$, $P<0.05)$ than controls. Furthermore, muscle triglyceride content was about $25 \%$ lower in WAT ${ }^{A^{Y} / a D g a t 1}{ }^{\prime} \rightarrow$ $A^{Y} / a$ Dgat $1^{+/+}$mice than in control mice $(14.7 \pm 2.1 \mathrm{vs}$. $20.4 \pm 2.1 \mathrm{mg} / \mathrm{g}$ tissue weight, $n=3, P<0.05)$.

In contrast, transplantation of $o b / o b$ Dgat $1^{-/}$WAT into $o b / o b$ (WAT ${ }^{o b / o b D g a t 1^{-/}} \rightarrow o b / o b$ Dgat $1^{+/+}$) mice fed a chow diet did not significantly affect their blood glucose concentrations after a glucose load (Figure 6c) or an insulin injection (Figure 6d). Chow-fed WAT $^{o b / o b D g a t 1^{-/-}} \rightarrow o b / o b$ Dgat $1^{+++}$and control mice also had similar mean body weights $(69.2 \pm 6.2$ vs. $66.4 \pm 3.9 \mathrm{~g}$, $n=3, P>0.05)$, total fat pad content $(12.9 \% \pm 0.6 \%$ vs.

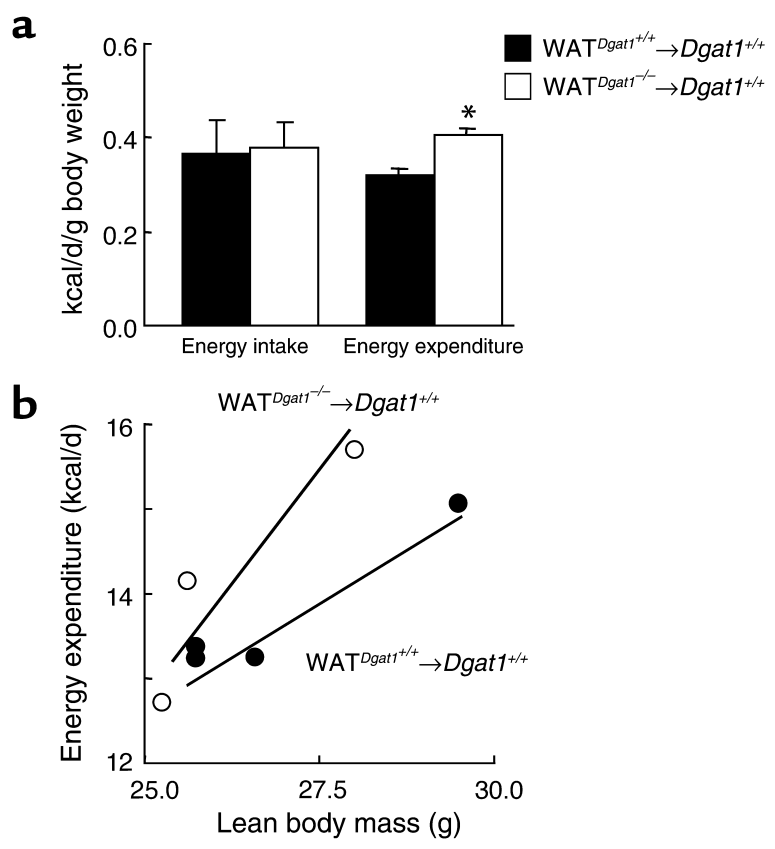

\section{Figure 4}

Effects of transplanted Dgat1-/- WAT on energy intake and expenditure in mice fed a high-fat diet. (a) Energy intake and expenditure in WAT $^{\text {Dat }} 1^{-/-} \rightarrow D$ gat $1^{+/+}$and WATDgat $1^{+/+} \rightarrow$ Dgat $1^{+/+}$mice. $n=3-4$ male mice per group. Error bars represent SEM. ${ }^{*} P<0.05$. (b) Relationship between energy expenditure and lean body mass in WAT $^{\text {Dgat }} 1^{-/-} \rightarrow$ Dgat $1^{+/+}$and WAT ${ }^{\text {gat }} 1^{+/+} \rightarrow D$ gat $1^{+/+}$mice. Regression lines for both groups are shown. 


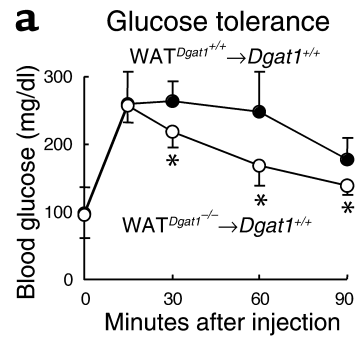

C
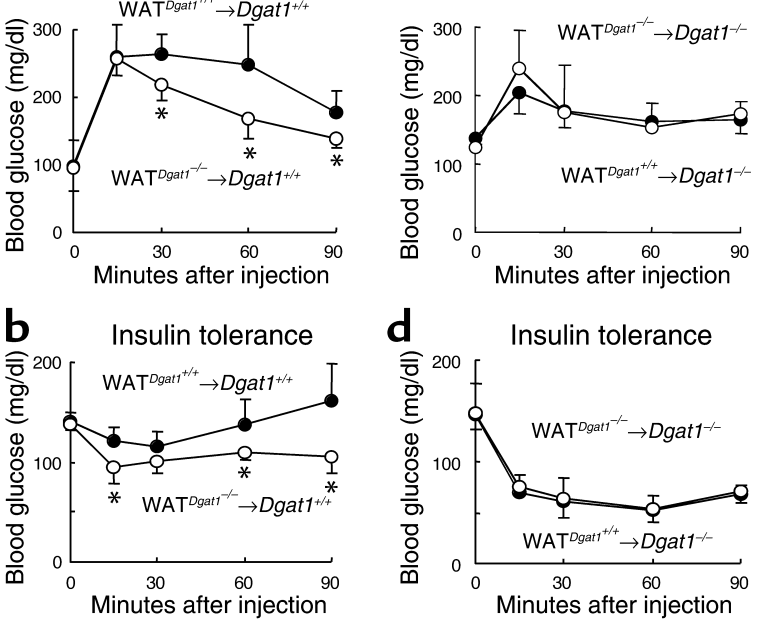

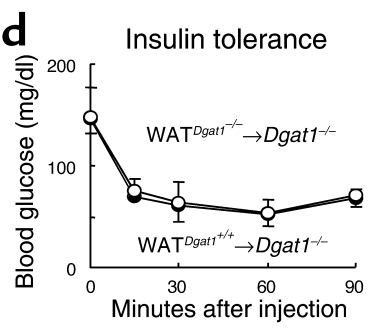

Figure 5

Effects of WAT transplantation on glucose metabolism. ( $\mathbf{a}$ and $\mathbf{b}$ ) Enhanced glucose disposal in WAT ${ }^{D a t 1^{-/-}} \rightarrow$ Dgat $1^{+/+}$mice. $n=5-6$ chow-fed male mice per group. (c and $\mathbf{d}$ ) Lack of effect of transplanted Dgat1 $1^{+/+}$WAT on glucose disposal in Dgat $1^{-/-}$mice. $n=4$ chow-fed male mice per group. ${ }^{*} P<0.05$.

$13.4 \% \pm 2.0 \%$ of total body weight, $n=3, P>0.05)$, and muscle triglyceride content $(7.0 \pm 2.3$ vs. $6.7 \pm 1.9 \mathrm{mg} / \mathrm{g}$ tissue weight, $n=3, P>0.05$ ).

Increased adiponectin mRNA expression in Dgat1-/- mice. The WAT transplantation results suggested that Dgat1 $1^{-1}$ WAT secretes factors that increase energy expenditure and enhance insulin sensitivity. We therefore examined the gene expression of several adipocytederived molecules in WAT of Dgat $1^{-/-}$mice. Dgat $1^{1^{--}}$and Dgat $1^{+/+}$mice had similar levels of expression of resistin and TNF- $\alpha$ (Figure 7a), molecules implicated in the development of obesity-induced insulin resistance $(6,7)$. In contrast, the mRNA levels of adiponectin, a molecule that enhances insulin sensitivity and fatty acid oxidation $(4,5,21)$, trended higher in chow-fed Dgat $1^{-/-}$mice than in chow-fed Dgat $1^{+/+}$mice, and the increase was statistically significant in $D g a t 1^{-/-}$mice fed a high-fat diet. DGAT1 deficiency also increased adiponectin expression twofold in $A^{Y} /$ a mice. Furthermore, 1 week after transplantation, adiponectin expression was twofold greater in the transplanted $A^{Y} / a$ Dgat1 $1^{-/-}$WAT than in the native $A^{Y} /$ a Dgat $1^{+/+}$WAT (Figure $7 \mathrm{~b}$ ), indicating that the increase in adiponectin mRNA expression in Dgat1 ${ }^{-/}$WAT was maintained after transplantation.

To determine the effects of leptin and DGAT1 deficiency on adiponectin expression, we measured adiponectin mRNA levels in $o b / o b$ mice. Adiponectin expression levels were low in $o b / o b D g a t 1^{+/+}$mice (Figure $7 c)$, consistent with previous findings (22). In contrast to the results in high-fat fed mice and $A^{Y} / a$ mice, DGAT1 deficiency had no effect on the low expression levels of adiponectin in $o b / o b$ mice. Five days of leptin infusion (6 or $12 \mu \mathrm{g} / \mathrm{d}$ ) significantly increased adiponectin expression in $o b / o b$ Dgat $1^{-/-}$mice but had a minimal effect on adiponectin expression in $o b / o b D g a t 1^{+/+}$mice.
To investigate whether leptin directly increases the expression of adiponectin in mice with an intact leptin pathway, we infused leptin subcutaneously into $D g a t 1^{-/-}$and $D g a t 1^{+/+}$mice. Leptin infusion $(12 \mu \mathrm{g} / \mathrm{d})$ did not increase the expression of adiponectin in either genotype (Figure 7d). These results suggest that leptin plays a permissive rather than a direct regulatory role in the expression of adiponectin.

Correlation of secreted-protein levels with $m R N A$ expression in Dgat1-/- WAT. To determine whether WAT mRNA expression correlated with the amounts of proteins secreted by $D$ gat1 $1^{-/}$WAT, we analyzed growth media conditioned by either $A^{Y} / a$ agat $1^{-/-}$or $A^{Y} / a$ Dgat $1^{+/+}$ WAT. Media conditioned by either $A^{Y} / a$ Dgat $1^{-/-}$or $A^{Y} / a$ Dgat $1^{+/+}$WAT had similar levels of total protein (Figure $8 \mathrm{a})$ and FFAs (Figure 8b). Medium conditioned by $A^{Y} / a$ Dgat1 $1^{-/}$WAT had less leptin than medium conditioned by $A^{Y} / a$ Dgat $1^{+/+}$WAT (Figure $8 c$ ). This result is consistent with our previous in vivo finding that Dgat $1^{-/-}$ WAT has decreased expression of leptin (16). TNF- $\alpha$ levels were similar in the two media (Figure 8d), whereas adiponectin levels were increased twofold in medium conditioned by $A^{Y} /$ a Dgat $1^{-/-}$WAT (Figure 8e).

Increased serum adiponectin levels in mice transplanted with Dgat1 $1^{-/}$WAT. To determine whether the ex vivo finding of increased adiponectin secretion by Dgat1-/WAT correlated with increased serum adiponectin levels in the transplant model, we measured serum adiponectin concentrations in $\mathrm{WAT}^{\text {Dgat } 1^{-/}} \rightarrow$ Dgat $1^{+/+}$ and WAT $^{\text {Dgat }}{ }^{+/+} \rightarrow D$ gat $1^{+/+}$mice fed a high-fat diet. After 2 weeks of high-fat feeding, serum adiponectin levels trended higher in $\mathrm{WAT}^{\text {Dgat1 }}{ }^{-/-} \rightarrow$ Dgat $1^{+/+}$mice than in WAT $^{\text {gat 1 }} 1^{+/+} \rightarrow$ Dgat $^{+/+}$mice (Figure $\left.9 a\right)$. After 5 weeks of high-fat feeding, the difference was statisti-
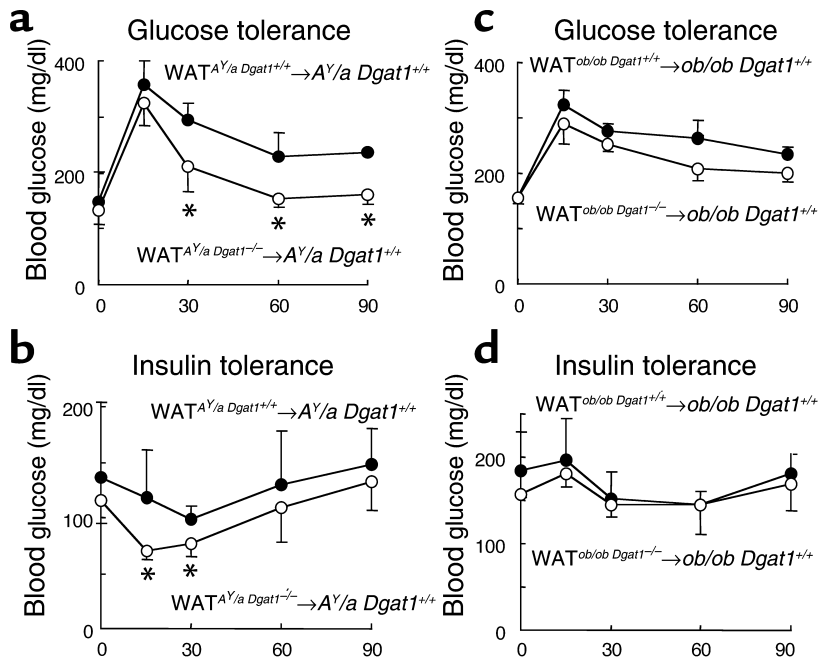

\section{Figure 6}

Metabolic effects of transplanted Dgat1 $1^{-/-}$WAT in $A^{Y} / a$ and $o b / o b$ mice. (a and $\mathbf{b}$ ) Enhanced glucose disposal in WAT ${ }^{A^{Y} / a \mathrm{aDat} 1^{-/-}} \rightarrow A^{Y} / a$ Dgat $1^{+/+}$mice. $n=4-5$ chow-fed male mice per group. (c and d) Lack of effect of transplanted ob/ob Dgat $1^{-/-}$WAT on glucose disposal in $o b / o b D$ gat $1^{+/+}$mice. $n=4$ chow-fed male mice per group. ${ }^{*} P<0.05$. 
a
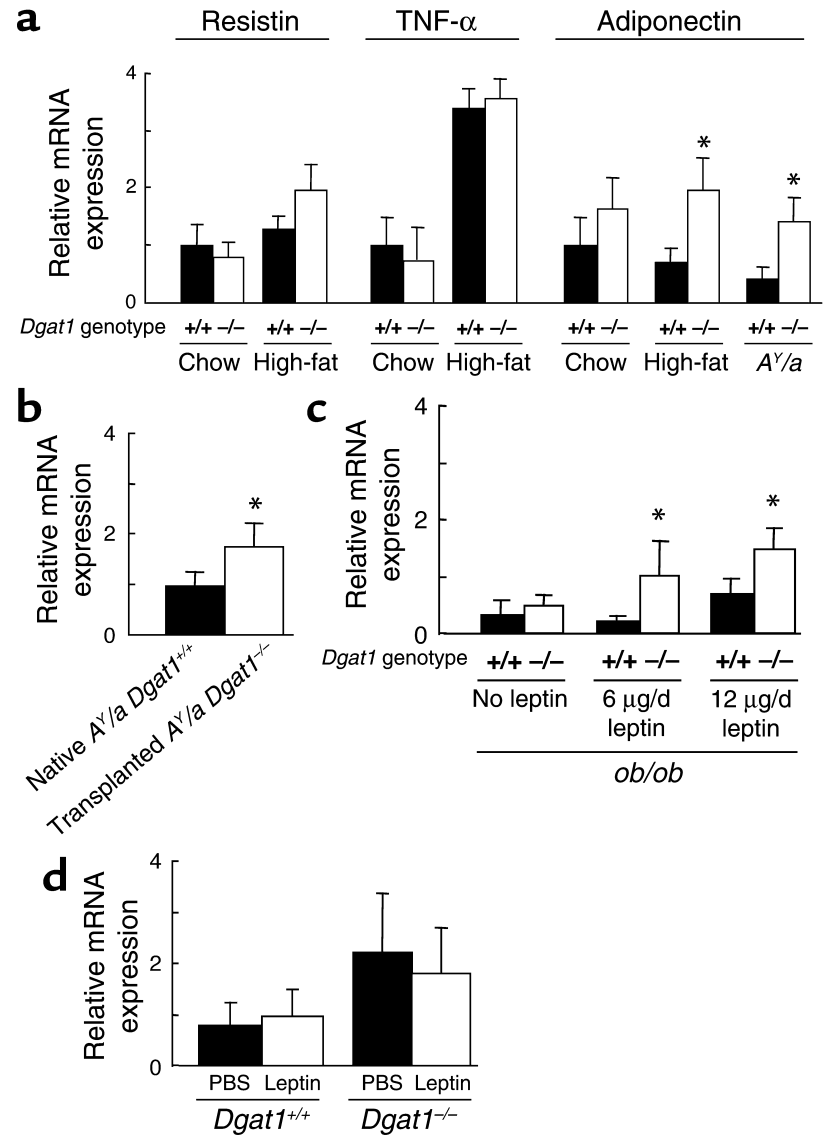

cally significant and persisted after 20 weeks of highfat feeding (Figure 9b).

Serum adiponectin and TNF- $\alpha$ levels in Dgat $1^{-/-}$mice. We measured serum levels of adiponectin and TNF- $\alpha$ in nontransplanted $D$ gat $1^{-/-}$mice. After 8 weeks of high-fat feeding, Dgat $1^{+/+}$, Dgat $^{+/-}$, and Dgat $1^{-/-}$mice had similar serum adiponectin levels (Figure 10a). However, after adjustment of these values for total fat pad weight $(1.0 \pm 0.3 \mathrm{~g}$ for $D$ gat $1^{-/-}, 1.7 \pm 0.3 \mathrm{~g}$ for $D g a t 1^{+/-}$, and $4.4 \pm 0.5 \mathrm{~g}$ for Dgat $1^{+/+}$mice, $n=5-7, P<0.05$ for all comparisons), Dgat 1 $1^{-/}$mice had the highest relative adiponectin levels, and $D g a t 1^{+/+}$mice had the lowest. We also examined serum adiponectin levels in chow-fed $A^{Y} / a$ mice. $A^{Y} / a$ Dgat $1^{+/+}$and $A^{Y} /$ a Dgat1 $1^{-/-}$mice had comparable serum adiponectin levels $(18.1 \pm 2.5 \mathrm{vs} .19 .3 \pm 4.5 \mu \mathrm{g} / \mathrm{ml}, n=5-6$, $P>0.05)$. However, $A^{Y} / a$ Dgat1 $1^{-/-}$mice had higher serum adiponectin levels than $A^{Y} /$ a Dgat $1^{+/+}$mice after adjustment for fat pad weight (Figure 10b).

In contrast to adiponectin, serum TNF- $\alpha$ levels were lower in Dgat $1^{-/-}$mice than in Dgat $1^{+/+}$mice after 8 weeks of high-fat feeding (Figure 10c). TNF- $\alpha$ levels also trended lower in $D g a t 1^{+/-}$mice than in Dgat $1^{+/+}$mice, although the difference was not statistically significant. After adjustment for total fat pad weight, however, all three groups of mice had similar relative TNF- $\alpha$ levels.

Because TNF- $\alpha$ may antagonize the actions of adiponectin (23), the serum adiponectin/TNF- $\alpha$ ratio may be an important determinant of energy metabo-

\section{Figure 7}

Expression of adipocyte-derived molecules. (a) Resistin, TNF- $\alpha$, and adiponectin expression in WAT of Dgat $1^{-/-}$mice. $n=7-10$ male mice per group. Error bars represent SEM. ${ }^{*} P<0.05$ vs. Dgat $1^{+/+}$mice fed a high-fat diet for 20 weeks. (b) Comparison of adiponectin expression in transplanted $A^{Y} / a$ Dgat1 $1^{-/-}$WAT and native $A^{Y} / a$ Dgat1 $1^{+/+}$ WAT obtained from transplanted mice. $n=4$ male mice per group. ${ }^{*} P<0.05$ vs. naive WAT. (c) Effect of leptin infusion on adiponectin expression in ob/ob Dgat1-/- mice. $n=3-5$ male mice per group. Expression of chow-fed Dgat $1^{+/+}$mice $=1.0 .{ }^{*} P<0.05$ vs. ob $/ o b$ $D$ gat $1^{+/+}$mice without leptin treatment. (d) Effect of leptin infusion on adiponectin expression in Dgat $1^{-I_{-}^{-}}$and Dgat $1^{+/+}$mice. $n=8-10$ male mice per group.

lism and systemic insulin sensitivity. The mean adiponectin/TNF- $\alpha$ ratio was twofold higher in highfat fed Dgat $1^{-/-}$mice than in high-fat fed $D$ gat $1^{+/+}$mice (2.4 vs. 1.2), correlating with their increased energy expenditure and enhanced insulin sensitivity.

\section{Discussion}

In this study, we show that the transplantation of Dgat $1^{-/}$WAT decreased adiposity and enhanced glucose disposal in $D g a t 1^{+/+}$mice. Our results suggest that DGAT1 deficiency alters the endocrine function of WAT in a manner that promotes obesity resistance and enhances insulin sensitivity. Although multiple secretory factors may be involved, we identified one
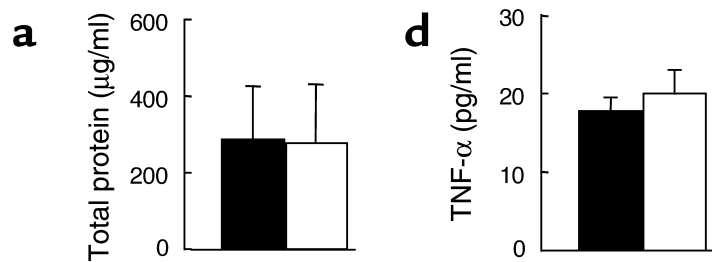

Dgat1 genotype +/+ -/-

Dgat1 genotype +/+ -/-

b

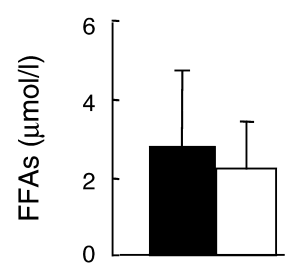

e



D

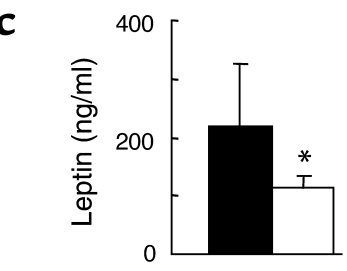

Dgat1 genotype +/+ -/-

Figure 8

Concentrations of adipocyte-derived factors in growth media conditioned by $A^{Y} / a$ Dgat $1^{-/-}$or $A^{Y} /$ a Dgat $1^{+/+}$WAT. (a) Total protein. (b) FFAs. (c) Leptin. (d) TNF- $\alpha$. (e) Adiponectin. $n=6-10$ mice per group. ${ }^{*} P<0.05,{ }^{*} P<0.01$. 
a



b

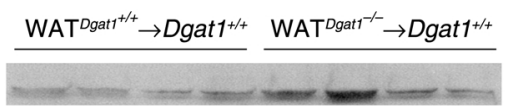

Figure 9

Increased serum adiponectin levels in WAT Dgat $^{1^{-/}} \rightarrow$ Dgat $1^{+/+}$mice fed a high-fat diet. (a) After 2 or 5 weeks of high-fat feeding. $n=4-5$ male mice per group. ${ }^{*} P<0.05$. (b) After 20 weeks of high-fat feeding. Each lane represents a serum sample from an individual mouse. The experiment was performed twice, and representative results are shown.

possible contributing factor - the increased production of adiponectin by Dgat $1^{-/-}$WAT. Our findings show that the technique of fat transplantation described by Gavrilova et al. (10) and Colombo et al. (24) can be used to study the metabolic effects of genetically engineered WAT.

The transplantation of Dgat1 $1^{-/}$WAT conferred several aspects of the DGAT1-deficiency phenotype on Dgat $1^{+/+}$mice. Chow-fed WAT Dgat $^{-/ /} \rightarrow$ Dgat $1^{+/+}$mice had enhanced glucose disposal. WAT Dgat $^{-\gamma_{-}} \rightarrow$ Dgat $1^{+/+}$mice gained less weight than control mice in response to a high-fat diet, similar to what occurs in Dgat $1^{-/-}$mice (12). WAT $^{\text {Dgat1 }}$-/- $_{\rightarrow} \rightarrow$ gat $^{+/+}$mice lost more weight during prolonged fasting and consumed more food than controls during refeeding, again mirroring the responses of $D g a t 1^{-/-}$mice (25). These findings, along with the results from the energy-balance studies, suggest that the transplantation of Dgat1-/- WAT confers obesity resistance primarily by increasing energy expenditure.

The transplantation of $D$ gat $1^{-/-}$WAT decreased adiposity and enhanced glucose disposal in $A^{Y} / a$ mice but had no apparent effects in $o b / o b$ mice. One possible explanation for this lack of effect is that the amount of transplanted WAT may be insufficient to overcome the severe obesity and insulin resistance in $o b / o b$ mice. Alternatively, the effects of DGAT1 deficiency on WAT endocrine functions may require leptin. This explanation would be consistent with our previous findings that the effects of DGAT1 deficiency on obesity resistance (16), increased expression of uncoupling protein $1(25,26)$, and sebaceous gland atrophy and hair loss (20) were present in $A^{Y} / a$ mice but not in $o b / o b$ mice. Our transplantation results suggest that DGAT1 deficiency alters the expression of adipocyte-derived factors that confer obesity resistance and enhance glucose disposal. The adipose tissue has been recognized as an important endocrine organ, secreting several molecules that modulate energy and glucose metabolism $(1,2)$. These molecules include adiponectin (also known as
Acrp30 [ref. 27], apM1 [ref. 28], and AdipoQ [ref. 22]), which enhances fatty acid oxidation and increases insulin sensitivity, and TNF- $\alpha$, which induces insulin resistance. Mice treated with adiponectin have decreased adiposity, decreased muscle triglyceride content, and increased insulin sensitivity $(4,5,21)$. Recent studies suggest that TNF- $\alpha$ suppresses adiponectin expression and antagonizes its effects on energy and glucose metabolism (23). Thus, the relative levels of adiponectin and TNF- $\alpha$ in the serum may be an important determinant of energy expenditure and systemic insulin sensitivity (23).

Several of our findings indicate that Dgat $1^{-/}$WAT produced more adiponectin than an equivalent amount of Dgat $1^{+/+}$WAT. These findings include the increased mRNA expression in Dgat $1^{-/-}$WAT, increased protein concentration in growth medium conditioned by $A^{Y} / a$ Dgat 1-/ WAT, and increased serum levels in WAT $^{\text {Dgat }} 1^{-/-} \rightarrow D$ gat $1^{+/+}$mice. These results imply that increased adiponectin secretion by transplanted Dgat1 ${ }^{-/-}$ WAT may contribute to the obesity resistance and enhanced glucose metabolism in $\mathrm{WAT}^{\text {Dgat }} 1^{--} \rightarrow$ Dgat $^{+/+}$
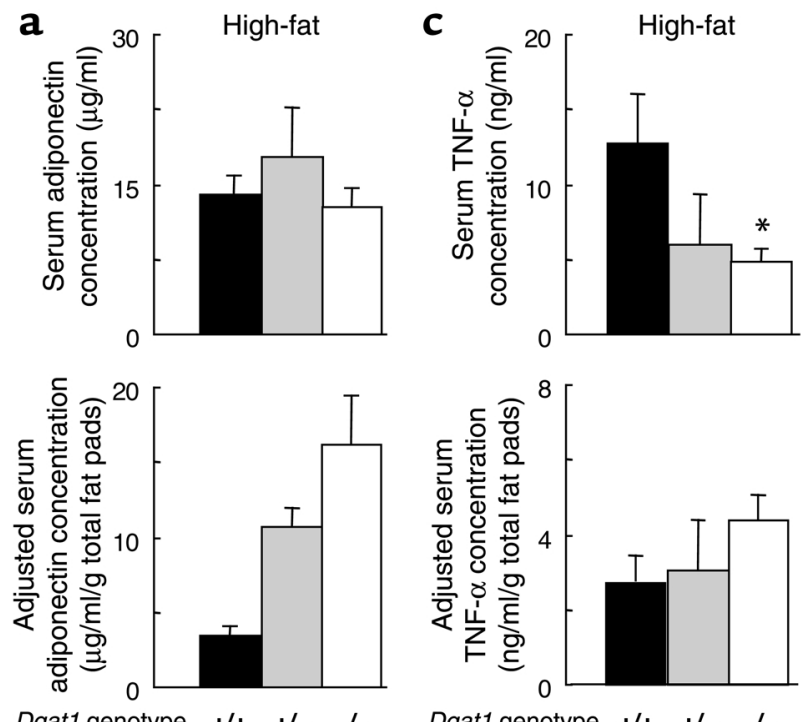

Dgat1 genotype +/+ +/- -/-

Dgat1 genotype +/+ +/- -/-



Figure 10

Serum adiponectin and TNF- $\alpha$ levels in Dgat $1^{-/-}$mice. (a) Serum adiponectin levels in $D g a t 1^{+/+}, D g a t 1^{+/-}$, and Dgat $1^{-/-}$mice fed a high-fat diet. $n=5-7$ male mice per group. $P<0.01$ for all comparisons for adjusted serum concentrations. (b) Adjusted serum adiponectin levels in $A^{Y} / a$ Dgat $1^{+/+}$and $A^{Y} / a$ Dgat $1^{-/-}$mice fed a chow diet. $n=5-6$ male mice per group. ${ }^{*} P<0.01$. (c) Serum TNF- $\alpha$ levels in Dgat $1^{-/-}$mice fed a high-fat diet. $n=5-7$ male mice per group. ${ }^{*} P<0.05 \mathrm{vs}$. Dgat $1^{+/+}$mice. 
mice. However, our data do not exclude the possibility that alterations in the expression of additional adipocyte-derived factors, including nonpeptides such as lysophosphatidic acid (29), may also contribute to the findings in $\mathrm{WAT}^{\text {Dgat } 1^{-/}} \rightarrow$ Dgat $1^{+/+}$mice.

Interestingly, despite increased adiponectin production by $D$ gat $1^{-/-}$WAT, serum adiponectin levels were similar in nontransplanted $D g a t 1^{-/-}$and $D g a t 1^{+/+}$mice. This lack of difference most likely reflects the 50\% reduction in adipose mass in Dgat $1^{-/-}$mice (12). The decreased adiposity also helps to explain the 50-60\% reduction in serum TNF- $\alpha$ levels in Dgat $1^{-/-}$mice, even though TNF- $\alpha$ expression was similar in $D g a t 1^{-/-}$and Dgat $1^{+/+}$WAT. Although Dgat $1^{-/-}$and Dgat $1^{+/+}$mice had similar serum levels of adiponectin, increased adiponectin action may still contribute to the increased energy expenditure (12) and enhanced insulin sensitivity (16) in $D$ gat $1^{-/-}$mice. Because of the decreased serum TNF- $\alpha$ levels in Dgat1 $1^{--}$mice, their adiponectin/TNF- $\alpha$ ratio was increased twofold. This increase may correlate with enhanced adiponectin action, or increased adiponectin sensitivity, in Dgat1-/mice. Such an effect would be similar to the effect of DGAT1 deficiency on increasing leptin sensitivity (16).

It is unclear how DGAT1 deficiency alters the endocrine functions of the adipocyte. Adiponectin expression is increased by insulin (30) and correlates inversely with adipocyte size (31). Two characteristics of $D g a t 1^{-/-}$mice, therefore, may contribute to their increased adiponectin expression: increased insulin sensitivity and small adipocyte size (16). It would be of interest to determine whether adiponectin expression is increased in other murine knockout models of obesity resistance, enhanced insulin sensitivity, and decreased adipocyte size (reviewed in ref. 11).

In conclusion, we show that the transplantation of Dgat $1^{-/-}$WAT confers obesity resistance and enhances glucose disposal in mouse models of obesity and insulin resistance. These results highlight the importance of WAT as an endocrine organ that regulates energy and glucose metabolism through secreted factors. In addition, our study demonstrates that fat transplantation may provide a useful tool for studying the endocrine and metabolic functions of genetically engineered WAT.

\section{Acknowledgments}

We thank M. Reitman and O. Gavrilova for advice on fat transplantation, J. Fruebis, S. Javorschi, and C. Vaisse for helpful discussions, B. Taylor for manuscript preparation, S. Ordway and G. Howard for editorial assistance, and M. Schambelan, R. Mahley, J. Herz, K. Weisgraber, and R. Streeper for comments on the manuscript. This work was supported by the NIH (K08-DK61363 to H.C. Chen, R01-DK26356 to R.H. Eckel, and R01-DK56084 to R.V. Farese), the Sandler Family Supporting Foundation, and the J. David Gladstone Institutes.

1. Flier, J.S. 1995. The adipocyte: storage depot or node on the energy information superhighway? Cell. 80:15-18.

2. Frühbeck, G., Gómez-Ambrosi, J., Muruzábal, F.J., and Burrell, M.A. 2001.
The adipocyte: a model for integration of endocrine and metabolic signaling in energy metabolism regulation. Am. J. Physiol. Endocrinol. Metab. 280:E827-E847.

3. Zhang, Y., et al. 1994. Positional cloning of the mouse obese gene and its human homologue. Nature. 372:425-432.

4. Yamauchi, T., et al. 2001. The fat-derived hormone adiponectin reverses insulin resistance associated with both lipoatrophy and obesity. Nat. Med. 7:941-946.

5. Berg, A.H., Combs, T.P., Du, X., Brownlee, M., and Scherer, P.E. 2001. The adipocyte-secreted protein Acrp30 enhances hepatic insulin action. Nat. Med. 7:947-953.

6. Hotamisligil, G.S., Shargill, N.S., and Spiegelman, B.M. 1993. Adipose expression of tumor necrosis factor- $\alpha$ : direct role in obesity-linked insulin resistance. Science. 259:87-91.

7. Steppan, C.M., et al. 2001. The hormone resistin links obesity to diabetes. Nature. 409:307-312.

8. Shimomura, I., et al. 1998. Insulin resistance and diabetes mellitus in transgenic mice expressing nuclear SREBP-1c in adipose tissue: model for congenital generalized lipodystrophy. Genes Dev. 12:3182-3194.

9. Moitra, J., et al. 1998. Life without white fat: a transgenic mouse. Genes Dev. 12:3168-3181.

10. Gavrilova, O., et al. 2000. Surgical implantation of adipose tissue reverses diabetes in lipoatrophic mice.J. Clin. Invest. 105:271-278.

11. Chen, H.C., and Farese, R.V., Jr. 2001. Turning WAT into BAT gets rid of fat. Nat. Med. 7:1102-1103.

12. Smith, S.J., et al. 2000. Obesity resistance and multiple mechanisms of triglyceride synthesis in mice lacking DGAT. Nat. Genet. 25:87-90.

13. Cases, S., et al. 1998. Identification of a gene encoding an acyl CoA:diacylglycerol acyltransferase, a key enzyme in triacylglycerol synthesis. Proc. Natl. Acad. Sci.U. S. A. 95:13018-13023.

14. Farese, R.V.,Jr., Cases, S., and Smith, S.J. 2000. Triglyceride synthesis: insights from the cloning of diacylglycerol acyltransferase. Curr. Opin. Lipidol. 11:229-234.

15. Cases, S., et al. 2001. Cloning of DGAT2, a second mammalian diacylglycerol acyltransferase, and related family members. J. Biol. Chem. 276:38870-38876.

16. Chen, H.C., et al. 2002. Increased insulin and leptin sensitivity in mice lacking acyl CoA:diacylglylcerol acyltransferase 1. J. Clin. Invest. 109:1049-1055. doi:10.1172/JCI200214672.

17. Halaas, J.L., et al. 1997. Physiological response to long-term peripheral and central leptin infusion in lean and obese mice. Proc. Natl. Acad. Sci. U. S. A. 94:8878-8883.

18. Fan, W., Boston, B.A., Kesterson, R.A., Hruby, V.J., and Cone, R.D. 1997. Role of melanocortinergic neurons in feeding and the agouti obesity syndrome. Nature. 385:165-168.

19. Chehab, F.F., Lim, M.E., and Lu, R. 1996. Correction of the sterility defect in homozygous obese female mice by treatment with the human recombinant leptin. Nat. Genet. 12:318-320.

20. Chen, H.C., Smith, S.J., Tow, B., Elias, P.M., and Farese, R.V., Jr. 2002. Leptin modulates the effects of acyl CoA:diacylglycerol acyltransferase deficiency on murine fur and sebaceous glands. J. Clin. Invest. 109:175-181. doi:10.1172/JCI200213880.

21. Fruebis, J., et al. 2001. Proteolytic cleavage product of 30-kDa adipocyte complement-related protein increases fatty acid oxidation in muscle and causes weight loss in mice. Proc. Natl. Acad. Sci. U. S. A. 98:2005-2010.

22. Hu, E., Liang, P., and Spiegelman, B.M. 1996. AdipoQ is a novel adipose-specific gene dysregulated in obesity. J. Biol. Chem. 271:10697-10703.

23. Maeda, N., et al. 2002. Diet-induced insulin resistance in mice lacking adiponectin/ACRP30. Nat. Med. 8:731-737.

24. Colombo, C., et al. 2002. Transplantation of adipose tissue lacking leptin is unable to reverse the metabolic abnormalities associated with lipoatrophy. Diabetes. 51:2727-2733.

25. Chen, H.C., Ladha, Z., and Farese, R.V., Jr. 2002. Deficiency of acyl CoA:diacylglycerol acyltransferase 1 increases leptin sensitivity in murine obesity models. Endocrinology. 143:2893-2898.

26. Chen, H.C., Ladha, Z., Smith, S.J., and Farese, R.V., Jr. 2003. Analysis of energy expenditure at different ambient temperatures in mice lacking DGAT1. Am. J. Physiol. Endocrinol. Metab. 284:E213-E218.

27. Scherer, P.E., Williams, S., Fogliano, M., Baldini, G., and Lodish, H.F. 1995. A novel serum protein similar to $\mathrm{C} 1 \mathrm{q}$, produced exclusively in adipocytes. J. Biol. Chem. 270:26746-26749.

28. Maeda, K., et al. 1996. cDNA cloning and expression of a novel adipose specific collagen-like factor, apM1 (adipose most abundant gene transcript 1). Biochem. Biophys. Res. Commun. 221:286-289.

29. Valet, P., et al. 1998. $\alpha_{2}$-Adrenergic receptor-mediated release of lysophosphatidic acid by adipocytes. A paracrine signal for preadipocyte growth. J. Clin. Invest. 101:1431-1438.

30. Halleux, C.M., et al. 2001. Secretion of adiponectin and regulation of apM1 gene expression in human visceral adipose tissue. Biochem. Biophys. Res. Commun. 288:1102-1107.

31. Yamauchi, T., et al. 2001. The mechanisms by which both heterozygous peroxisome proliferator-activated receptor $\gamma(\mathrm{PPAR} \gamma)$ deficiency and PPAR $\gamma$ agonist improve insulin resistance. J. Biol. Chem. 276:41245-41254. 